\title{
Polarization Waves in Nonlinear Fiber Optics.
}

\author{
V. V. Kozlov*,† and S. Wabnitz* \\ ${ }^{*}$ Department of Information Engineering, Università degli Studi di Brescia, Via Branze 38, 25123 Brescia, Italy \\ ${ }^{\dagger}$ Department of Physics, St.-Petersburg State University, Petrodvoretz, St.-Petersburg, 198504, Russia
}

\begin{abstract}
The subject of this contribution is the study of the nonlinear interaction of two quasi-monochromatic beams in a Kerr medium (e.g., an optical fiber) in the counter-propagating 1-D geometry. As shown by Zakharov and Mikhailov in Ref. [1], this problem is exactly integrable if the medium has infinite extension. However, very little is known when the medium is bounded from both sides, and boundary conditions are set at both boundaries. We study this case numerically and demonstrate that the soliton, which is stable in the infinite medium, becomes unstable when the medium is finite. We show that instead of the soliton, a so-called polarization attractor is a stable solution of the governing equations with an extremely broad basin of attraction. Moreover, we apply our findings to the practical problem of the design of nonlinear polarizers for high-speed telecom applications.
\end{abstract}

Keywords: polarization domain wall solitons, instability, attractors

PACS: $42.25 . J a, 42.65 . T g$, 42.81.Gs, 42.81.Dp

\section{INTRODUCTION.}

The impressive progress achieved over last two decades in the field of nonlinear fiber optics may create the delusive impression that the field is exhausted, hence it cannot deliver interesting physics anymore. This impression is based on the fact that most effects in nonlinear fibers are described by the well-studied scalar nonlinear Schrödinger equation (NLSE). In some more rare cases the scalar model is extended to a vector one, which permits to include in the picture the nonlinear dynamics of both polarization components of a light beam. Yet more rare studies extend this vectorial description to the interaction of two beams. Finally, the number of studies which consider the two-beam vector interaction in the counter-propagating geometry with boundary conditions set at both ends of the medium can be counted by the fingers on one hand. However, this level of complication is not a mental invention by the present authors. Quite to the opposite, it is the experiment which drives the interest in such model. Such experiments are conceptually very simple, albeit practically appealing. One uses a short span of fiber (usually, a few kilometers long), feeds two powerful beams from both ends, and follows the state of polarization (SOP) of each beam at the corresponding output end. When one beam (pump) is initially fully polarized and the other beam (signal) is initially unpolarized, the nonlinear interaction of the two beams in the Kerr medium results in the efficient repolarization of the signal beam. Saying this differently, the initially unpolarized signal beam becomes highly polarized towards its output end. Such dynamics allows one to construct a polarizer - the device which transform unpolarized light into highly polarized light. Driven by these practical considerations, we will derive a set of equations governing the propagation of counter-propagating beams in the medium with cubic nonlinearity for different types of fibers, and numerically study the behavior of its solutions.

\section{MODEL}

First of all, we note that fibers may provide significantly more complicated responses to applied light beams than predicted by a simple scalar NLSE. The reason for this complication is the nontrivial behavior of the SOP of the beams. The underlying physical mechanism for this dynamics is the unavoidable birefringence of realistic fibers, because the circular symmetry of the fiber core is broken owing to imperfections of the manufacturing process, so that the fiber core becomes slightly elliptic. Moreover, this ellipticity changes stochastically along the fiber length. Overall, telecom fibers are not isotropic but randomly birefringent: This type of fibers dominates the market. There are also speciality fibers, such as highly birefringent (Hi-Bi) fibers and spun fibers. Propagation of light in each of these types of fibers is described by its own set of equations. Without going into the details of the derivation, and simply referring to earlier studies Refs. [2, 3], we just report here the final result. It is remarkable that isotropic, randomly birefringent,

Numerical Analysis and Applied Mathematics ICNAAM 2011

AIP Conf. Proc. 1389, 697-700 (2011); doi: 10.1063/1.3636826

(C) 2011 American Institute of Physics 978-0-7354-0956-9/\$30.00 
$\mathrm{Hi}-\mathrm{Bi}$, and spun fibers, all are described by the single generic model:

$$
\partial_{\xi} \mathbf{S}^{+}=\gamma \mathbf{S}^{+} \times \mathbf{J}_{s} \mathbf{S}^{+}+\gamma \mathbf{S}^{+} \times \mathbf{J}_{x} \mathbf{S}^{-} \quad \partial_{\eta} \mathbf{S}^{-}=\gamma \mathbf{S}^{-} \times \mathbf{J}_{s} \mathbf{S}^{-}+\gamma \mathbf{S}^{-} \times \mathbf{J}_{x} \mathbf{S}^{+} .
$$

Here $\gamma$ is the Kerr coefficient, $\xi=(c t+z) / 2, \eta=(c t-z) / 2, \times$ denotes vector product, $\mathbf{J}_{s}$ and $\mathbf{J}_{x}$ are diagonal $3 \times 3$ self-polarization and cross-polarization matrices, correspondingly. As usual, the SOPs of the light beams are described by a three-component Stokes vector, whose tip conveniently represents the SOP as a point on the Poincaré sphere. The Stokes vector components are defined in terms of the original electric fields $E_{x}^{ \pm}$and $E_{y}^{ \pm}$(where plus sign is related to the field propagating in the positive direction of the $z$ axis, while minus sign - to the field propagating in the opposite direction) according to the following convention: $S_{1}^{ \pm}=E_{x}^{ \pm} E_{y}^{ \pm *}+$ c.c, $S_{2}^{ \pm}=i E_{x}^{ \pm} E_{y}^{ \pm *}-$ c.c, and $S_{3}^{ \pm}=\left|E_{x}^{ \pm}\right|^{2}-\left|E_{y}^{ \pm}\right|^{2}$.

For isotropic fibers $\mathbf{J}_{s}=\frac{1}{3} \operatorname{diag}(-2,0,-2)$ and $\mathbf{J}_{x}=\frac{2}{3} \operatorname{diag}(-2,0,-2)$; for randomly birefringent fibers $\mathbf{J}_{s}=$ $\operatorname{diag}(0,0,0)$ and $\mathbf{J}_{x}=\frac{8}{9} \operatorname{diag}(-1,1,-1)$; for Hi-Bi fibers $\mathbf{J}_{s}=\frac{1}{3} \operatorname{diag}(0,0,-1)$ and $\mathbf{J}_{x}=\frac{2}{3} \operatorname{diag}(1,-1,-2)$; for spun fibers $\mathbf{J}_{s}=\frac{1}{3} \operatorname{diag}\left(0,0,2 \sin ^{2} \phi-\cos ^{2} \phi\right)$ and $\mathbf{J}_{x}=\frac{2}{3} \operatorname{diag}\left(\cos ^{2} \phi,-\cos ^{2} \phi,-2 \cos ^{2} \phi\right)$, where $\phi$ characterizes the speed with which the fiber is spun during the manufacturing process.

It is known (see Ref. [1]) that the system (1) is integrable, providing that the self-polarization matrix vanishes: $\mathbf{J}_{s}=\operatorname{diag}(0,0,0)$. In this study we only concentrate on this particular case. This means that we consider randomly birefringent fibers with $\mathbf{J}_{x}=\frac{8}{9} \operatorname{diag}(-1,1,-1)$ and spun fibers with the special choice of $\phi=\phi_{0}$, such that $2 \sin ^{2} \phi_{0}=$ $\cos ^{2} \phi_{0}$, and therefore $\mathbf{J}_{x}=\frac{2}{3} \cos ^{2} \phi_{0} \operatorname{diag}(1,-1,-2)$. These two situations are dramatically different not only from the viewpoint of their different technological origin, but also from the viewpoint of the set of stable solutions that they possess.

\section{POLARIZATION DOMAIN WALL SOLITONS}

Since equations in (1) in the absence of self-interaction terms are integrable, they allow for the existence of soliton solutions. Following the spirit of the work Ref. [1], and the identity of these equations with the model of an anisotropic chiral field, these solutions are usually called domain wall solitons (DWS). For the particular case of spun fibers $\left[\mathbf{J}_{x}=\frac{2}{3} \cos ^{2} \phi_{0} \operatorname{diag}(1,-1,-2)\right]$, the soliton Stokes components read as

$$
\begin{aligned}
& S_{1}^{ \pm}(z)= \pm \frac{1}{3} \operatorname{sech}\left(\frac{z-z_{0}}{\sqrt{2} L_{n l}}\right), \\
& S_{2}^{ \pm}(z)=\mp \tanh \left(\frac{z-z_{0}}{\sqrt{2} L_{n l}}\right), \\
& S_{3}^{ \pm}(z)= \pm \frac{2}{3} \operatorname{sech}\left(\frac{z-z_{0}}{\sqrt{2} L_{n l}}\right),
\end{aligned}
$$

see $[4,6]$. Here $L_{n l}=\left(\frac{2}{3} \cos ^{2} \phi_{0} \gamma S_{0}\right)^{-1}$ has the meaning of a characteristic nonlinear length. This solution is a standing wave soliton, i.e. it represents the distribution of the SOP (polarization walls) of the light beams "frozen" inside the medium. Such standing-wave soliton can only be excited when the powers of the two beams are equal: $S_{0}^{+}=S_{0}^{-}$, where $S_{0}^{ \pm}=\left[\left(S_{1}^{ \pm}\right)^{2}+\left(S_{2}^{ \pm}\right)^{2}+\left(S_{3}^{ \pm}\right)^{2}\right]^{1 / 2}$. The family of soliton solutions is broader, including in particular the moving solitons that appearing in the case of different beam powers: $S_{0}^{+} \neq S_{0}^{-}$. The moving solitons are not of interest to us here.

Strictly speaking, a soliton in the form of Eqs. (2)-(4) is only a solution for the case of an unbounded medium. At this point we ask the question, whether this soliton still exists when the medium is bounded from both sides, and proper boundary conditions (compatible with the soliton tails) are set at the two boundaries. We answer this question in the following section.

\section{INSTABILITY OF THE DOMAIN WALL SOLITONS}

We performed the following numerical experiment. We have set the boundary and initial conditions as demonstrated on the top left panel of Fig. 1. These conditions are close to the soliton solution which is given in Eqs. (2)-(4). Indeed, it is found that at some later time the soliton is formed in the center of the medium. However, in the course of time the soliton starts moving towards the left boundary, as seen on the bottom left panel. The closer it approaches the boundary, the higher its speed. This accelerated motion finally results in the break-down of the soliton when it hits the boundary. 

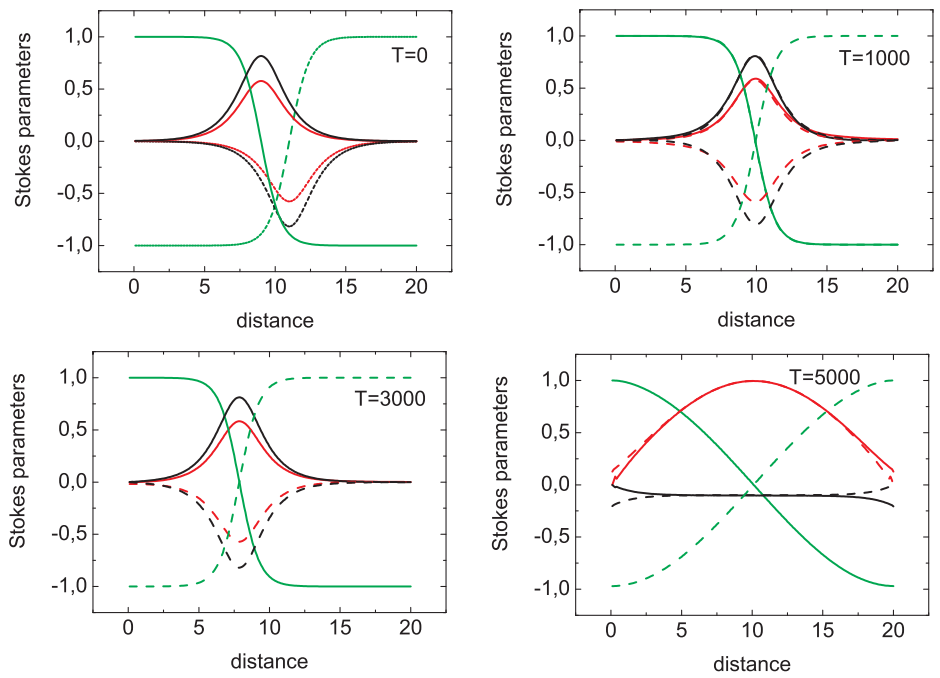

FIGURE 1. Formation and breakdown of polarization domain wall solitons. In all plots we show the Stokes parameters of forward and backward beams as a function of distance inside the medium of total length $L=20 L_{n l}$. Top left: input profiles. Top right: the result of evolution at $T=1000$, clearly demonstrating the formation of the soliton. The analytical soliton shape given by Eqs. (2)(4) is superimposed on the numerically generated shapes. The two sets of the curves are visually indistinguishable. Bottom left: snapshot of the evolution at $T=3000$ demonstrating the motion of the soliton towards the left boundary. Bottom right: shapes at $T=5000$ demonstrating the ultimate result of the evolution towards the steady-state in the form of a polarization attractor. The curves are designated as: Stokes parameters of the forward (solid) and backward (dashed) beam: $S_{1}^{ \pm}$(red), $S_{2}^{ \pm}$(green), $S_{3}^{ \pm}$(black). Time $T$ is measured in units $L_{n l} / c$. Note that the round-trip time between the boundaries is $T=40$.

Then, a new polarization pattern quickly forms, see bottom right panel. We name this pattern polarization attractor. To the best of our knowledge, the notion of polarization attractor was first introduced in the study of the phenomenon of polarization attraction in isotropic fibers in Ref. [7]. For this particular choice of boundary conditions, we always observed an evolution towards the formation of a polarization attractor, independently of the initial conditions.

From the above numerical experiment we conclude that the soliton is unstable in a bounded medium. The stable pattern is the polarization attractor. The qualitative difference between the two solutions is in the degree of their spatial extension. The soliton is the structure well-localized inside the medium, without any reference to the boundaries. In contrast, the polarization attractor is delocalized structure extending from one boundary to the other, thus occupying the entire medium.

Our study indicates an interesting example, when the soliton is unstable, though the underlying system is integrable. For such systems there is a clear need in the development of new methods for finding the stable solutions - polarization attractors. It is also important to extend the presented analysis to different forms of the cross-polarization matrix $\mathbf{J}_{x}$, and check whether soliton solutions are also unstable in these cases, or if in some cases they may remain stable.

\section{APPLICATION ASPECTS OF THE MODEL}

The model described by the set of equations in (1) has been used to study the phenomenon of polarization attraction in optical fibers, see Refs. [8, 9, 10, 11]. Briefly speaking, this phenomenon arises when one the beams (pump) is fully polarized (its SOP is a point on the Poincare sphere), while the other beam (signal) is unpolarized, that is its SOP changes stochastically with time (the tip of its Stokes vector randomly covers the entire Poincaré sphere). While propagating from the input boundary towards the output, the signal beam gradually acquires an high degree of polarization. This means that unpolarized light is converted into highly polarized light. A medium which is pumped by the powerful beam works as a polarizer for the signal beam, which also must be relatively intense: $S_{0}^{+} \sim S_{0}^{-}$. This effect holds much promise for useful telecom applications by enabling ultrafast all-optical polarization control of a bit stream (a sequence of short light pulses). An example demonstrating how the polarizer works is shown in Fig. 2. Given that initially the Stokes vector of the signal beam uniformly covers the Poincaré sphere, the corresponding 


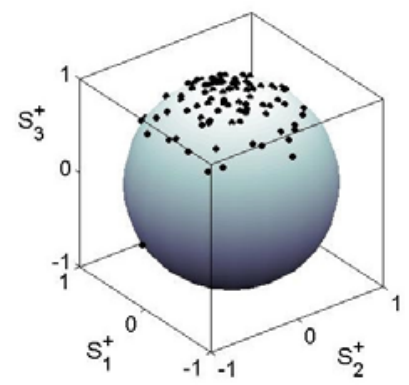

FIGURE 2. $N=110$ points on the Poincare sphere illustrating the output SOPs of the signal beam corresponding to an input distribution of $N=110$ points uniformly distributed over the Poincaré sphere. Here the input SOP of the pump beam is $(0.01,0.01,-0.9999)$. The pump power is $S_{0}^{-}=5.5 S_{0}^{+}$.

output distribution appears to be localized around a localized spot.

It is clear that polarization attractors, which form stable steady-state solutions of the model equations, play a key role in the polarization attraction phenomenon. Moreover, our numerical simulations show (see Ref. [12]) that in some cases periodic solutions appear instead of steady-state solutions. The rich variety of solutions that are observed for the model of Eqs. (2)-(4) indicates that the methods of Nonlinear Dynamics can be quite useful and appropriate for the study of this system. This may lead the way to a better understanding of the physical mechanism which is underlying the polarization attraction phenomenon.

\section{CONCLUSION}

We studied an integrable system of equations which exhibits stable soliton solutions in the case of an indefinitely long medium. On the other hand we have shown that when the medium is finite, the soliton loses its stability. Instead of the soliton, which is intrinsically localized, the stable solution of the model equations is the polarization attractor, which is a solution that extends over the entire medium. Polarization attractors, being stationary solutions, do not exhaust the variety of all stable solutions. Equally important are periodic solutions. We performed these studies with an eye towards the potential application of the physical model to the design of efficient all-optical polarizers for telecom networks.

\section{ACKNOWLEDGMENTS}

This work was carried out with support from the PRIN2008 project "Nonlinear cross-polarization interactions in photonic devices and systems".

\section{REFERENCES}

1. V. E. Zakharov and A. V. Mikhailov, JETP Letters 45, No. 6, 349 (1987).

2. V. V. Kozlov, Javier Nuño, and S. Wabnitz, J. Opt. Soc. Am. B 28, No. 1, pp. 100 (2011).

3. V. V. Kozlov and S. Wabnitz, Opt. Lett. 35, No. 23, 3949 (2010).

4. S. Wabnitz, Opt. Lett. 34, 908 (2009).

5. S. Wabnitz, IEEE Photonics Technol. Lett. 21, 875 Û877 (2009).

6. V. V. Kozlov and S. Wabnitz, Lett. Math. Phys. 96, 405 (2011).

7. S. Pitois, A. Picozzi, G. Millot, H. R. Jauslin, and M. Haelterman, Europhys. Lett. 70, 88 (2005).

8. S. Pitois, G. Millot, and S. Wabnitz, Phys. Rev. Lett. 81, 1409 (1998).

9. S. Pitois, G. Millot, and S. Wabnitz, S., J. Opt. Soc. Am. B 18, 432 (2001).

10. S. Pitois, J. Fatome, and G. Millot, Opt. Express 16, 6646 (2008).

11. J. Fatome, S. Pitois, P. Morin, and G. Millot, Opt. Express 18, 15311 (2010).

12. V. V. Kozlov, J. Fatome, P. Morin, S. Pitois, G. Millot, and S. Wabnitz, J. Opt. Soc. Am. B 28, No. 8, 1782 (2011). 